\title{
Glucocorticosteroids Differentially Regulate MMP-9 and Neutrophil Elastase in COPD
}

\author{
Ross Vlahos ${ }^{1}$, Peter A. B. Wark ${ }^{2}$, Gary P. Anderson ${ }^{1}$, Steven Bozinovski ${ }^{1 *}$ \\ 1 Department of Pharmacology, The University of Melbourne, Victoria, Australia, 2 Respiratory Medicine HMRI, John Hunter Hospital, Newcastle, NSW, Australia
}

\begin{abstract}
Background: Chronic Obstructive Pulmonary Disease (COPD) is currently the fifth leading cause of death worldwide. Neutrophilic inflammation is prominent, worsened during infective exacerbations and is refractory to glucocorticosteroids (GCs). Deregulated neutrophilic inflammation can cause excessive matrix degradation through proteinase release. Gelatinase and azurophilic granules within neutrophils are a major source of matrix metalloproteinase (MMP)-9 and neutrophil elastase (NE), respectively, which are elevated in COPD.

Methods: Secreted MMP-9 and NE activity in BALF were stratified according to GOLD severity stages. The regulation of secreted NE and MMP-9 in isolated blood neutrophils was investigated using a pharmacological approach. In vivo release of MMP-9 and NE in mice exposed to cigarette smoke (CS) and/or the TLR agonist lipopolysaccharide (LPS) in the presence of dexamethasone (Dex) was investigated.

Results: Neutrophil activation as assessed by NE release was increased in severe COPD (36-fold, GOLD II vs. IV). MMP-9 levels (8-fold) and activity (21-fold) were also elevated in severe COPD, and this activity was strongly associated with BALF neutrophils $(r=0.92, p<0.001)$, but not macrophages $(r=0.48, p=0.13)$. In vitro, release of NE and MMP-9 from fMLP stimulated blood neutrophils was insensitive to Dex and attenuated by the PI3K inhibitor, wortmannin. In vivo, GC resistant neutrophil activation (NE release) was only seen in mice exposed to CS and LPS. In addition, GC refractory MMP-9 expression was only associated with neutrophil activation.

Conclusions: As neutrophils become activated with increasing COPD severity, they become an important source of NE and MMP-9 activity, which secrete proteinases independently of TIMPs. Furthermore, as NE and MMP-9 release was resistant to GC, targeting of the PI3K pathway may offer an alternative pathway to combating this proteinase imbalance in severe COPD.
\end{abstract}

Citation: Vlahos R, Wark PAB, Anderson GP, Bozinovski S (2012) Glucocorticosteroids Differentially Regulate MMP-9 and Neutrophil Elastase in COPD. PLoS ONE 7(3): e33277. doi:10.1371/journal.pone.0033277

Editor: Dominik Hartl, University of Tübingen, Germany

Received December 1, 2011; Accepted February 13, 2012; Published March 7, 2012

Copyright: (c) 2012 Vlahos et al. This is an open-access article distributed under the terms of the Creative Commons Attribution License, which permits unrestricted use, distribution, and reproduction in any medium, provided the original author and source are credited.

Funding: This work was supported by funding from National Health and Medical Research Council of Australia. The funders had no role in study design, data collection and analysis, decision to publish, or preparation of the manuscript.

Competing Interests: The authors have declared that no competing interests exist.

*E-mail: bozis@unimelb.edu.au

\section{Introduction}

Proteinases generated within the lung environment regulate many physiological processes during infection, inflammation and subsequent tissue repair. Innate immune cells including macrophages and neutrophils are a major source of matrix metalloproteinase-9 (MMP-9) and neutrophil elastase (NE), which are central to these processes. In the normal setting, anti-proteinases such as $\alpha 1$-antitrypsin $(\alpha 1-\mathrm{AT})$, secretory leukoprotease inhibitor (SLPI) and tissue inhibitor of metalloproteinases (TIMPs) are in excess and provide an anti-proteinase screen to prevent deleterious effects [1]. In lung diseases such as chronic obstructive pulmonary disease (COPD) there is an imbalance leading to excessive proteinase activity that can cause host tissue damage [1]. Activated neutrophils release serine proteinases including NE, Proteinase-3 (PR-3) and MMPs including MMP-9 and MMP-8, which have been shown to be elevated in COPD (reviewed in [2]). COPD is primarily caused by cigarette smoke exposure and is already the fifth leading cause of death worldwide [3]. As the disease progresses, increased susceptibility to respiratory virus and/or bacteria promote acute exacerbations (AECOPD) that further amplifies inflammation [4,5]. Glucocorticosteroids (GCs) are a mainstay for current AECOPD management, although their efficacy in COPD is suboptimal as neutrophilic inflammation persists following GC treatment [6].

Elevated MMP-9 levels in COPD are related to sputum neutrophil numbers to suggest that this cell type is a major source $[7,8]$. Neutrophils contain tertiary gelatinase granules formed at later stages of myelopoiesis that act as a major reservoir for the rapid exocytosis of MMP-9 [9]. This can result in increased local proteolytic activity because unlike other mononuclear leukocytes, this cell type does not express the inhibitor, TIMP1 [10]. MMP-9 degrades collagen, elastin and gelatin and its levels inversely correlate with airflow obstruction [7]. Extracellular matrix derived N-acetyl Pro-Gly-Pro (Ac-PGP) also augments MMP-9 release from neutrophils [11]. NE is abundant in primary azurophil granules formed during the early stages of myelopoiesis and is secreted during neutrophil degranulation [9]. Free NE activity 
may also accumulate in airways from necrotic neutrophils that release their intracellular content. NE activity is elevated in COPD and the degree of NE localised to lung elastic fibers correlates with the degree of emphysema [12]. NE can degrade extracellular matrix components including elastin, collagens I-IV and fibrinogen and the genetic deficiency in $\alpha 1-\mathrm{AT}$ is associated with early onset pan lobular emphysema [13]. Serine proteinases such as NE are considered to be at the apex of the proteinase hierarchy in airways as they have the ability to activate MMPs including MMP9 via cleavage of pro-MMP-9 into active-MMP-9 [14]. Furthermore, NE preferentially degrades TIMP1 that is bound to MMP9, thereby liberating active MMP-9 [15]. NE can also activate the inflammatory NFאB pathway via a Toll-Like Receptor-4 (TLR-4) dependent manner and promote an acute inflammatory response [16]. The promoter of MMP-9 also contains an NFкB binding site that promotes its expression [17].

Here, we have directly measured secreted MMP-9 and NE in BAL-fluid (BALF) from a COPD cohort stratified according to GOLD stages and have shown that NE and MMP-9 activity increase with disease severity. In addition, neutrophils were strongly related to both proteinases in severe COPD. We hypothesised that neutrophils are an important source of NE and MMP-9 that is insensitive to GCs. To examine this, we have assessed their release from isolated blood neutrophils in the presence of pharmacological inhibitors and in an in vivo inflammatory model that combines CS exposure and acute inflammation (LPS).

\section{Results}

\section{Airway neutrophils release MMP-9 and NE in COPD}

COPD severity was classified as moderate (stage II, $n=9(31 \%)$ ), severe (stage III, $\mathrm{n}=9(31 \%)$ ), and very severe (stage IV, $\mathrm{n}=11(38 \%)$ ) according to GOLD criteria as previously described [18]. Inhaled corticosteroid (ICS) daily usage increased across the severity grades as summarised in Table 1. Neutrophil BALF numbers increased with disease severity (GOLD II; mean 0.18 (range 0.04-0.45), GOLD III; $1.12(0.03-8.5)$ and GOLD IV; $1.83(0.05-13.4)$ neutrophils $\left(\times 10^{6}\right.$ per $\mathrm{mL}$ ) with a significant increase observed in GOLD IV vs. GOLD II $(\mathrm{p}<0.05$, Figure 1A). NE activity also increased with disease severity (GOLD II; mean 0.016 (range 0.007-0.04), GOLD III; 0.25 $(0.002-1.25)$ and GOLD IV; $0.57(.007-1.31)$ with a significant increase observed in GOLD IV vs. GOLD II $(p<0.05$, Figure 1B). There was a positive correlation between neutrophil BALF numbers and NE activity (Figure 1C; $r=0.65, p<0.001$ ).

\section{Increased MMP-9 is associated with increased neutrophilic inflammation in severe COPD}

Gelatin zymography of COPD BALF identified major bands of activity within the $90 \mathrm{kDa}$ range, which is consistent with the molecular weight of pro $(92 \mathrm{kDa})$ and active (82 kDa) MMP-9 (Figure 2A). Total MMP-9 (pro and active forms) in BALF was next measured by quantitative ELISA. The expression of MMP-9 increased with COPD severity (GOLD II; mean 137 (range 17.7301), GOLD III; 833 (50-5360) and GOLD IV; 1127 (88.6-8820) $\mathrm{ng} / \mathrm{mL}$ with a significant increase observed in GOLD IV vs. GOLD II $(p<0.05$, Figure 2B). Active MMP-9 quantified using flourescein conjugated gelatin as a substrate also showed an increase in MMP-9 activity with COPD severity (GOLD II; mean 0.43 (range $0.21-$ 0.81), GOLD III; $4.78(0.21-30.4)$ and GOLD IV; $8.96(0.17-$ 27.4) $\times 10^{4} \mathrm{RFU}$, with a significant increase observed in GOLD IV vs. GOLD II $(p<0.05$, Figure $2 \mathrm{C})$. There was a strong correlation between MMP-9 expression and gelatinase activity, confirming MMP-9 as the major gelatinase enzyme in severe COPD BALF (Figure 2D; $\mathrm{r}=00.86, p<0.001$ ). There was also a strong association between BALF neutrophils and MMP-9 expression (Figure 3A, $\mathrm{r}=0.89, p<0.001$ ) and activity (Figure $3 \mathrm{~B}, \mathrm{r}=0.92, p<0.001$ ) in GOLD IV COPD. BALF macrophages and MMP-9 expression (Figure 3C, $\mathrm{r}=0.38, p=0.25$ ) and activity (Figure 3D, $\mathrm{r}=0.48$, $p=0.13)$ were not significantly associated in severe COPD.

\section{Stimulated blood neutrophils rapidly release MMP-9 and NE in a GC refractory manner}

Isolated blood neutrophils stimulated with fMLP rapidly release (20 min) NE (Figure 4A) and MMP-9 (Figure 4B) in a Dex resistant manner. Pretreatment for $15 \mathrm{~min}$ prior to fMLP stimulation with the PI3K inhibitor, wortmannin, blocked release of NE and MMP-9 by $\sim 50 \%$ (Figure $4 \mathrm{C} \& \mathrm{D}$ ), whereas the MAPK inhibitors (U0126; Erk pathway and SB203580; p38 pathway) were only effective in reducing MMP-9 release by $22 \%$ and $42 \%$ respectively (Figures $4 \mathrm{C} \& \mathrm{D}$ ).

\section{CS promotes GC resistant airway inflammation}

Intraperitoneal administration of Dex (20 $\mu \mathrm{g}$ per day) over four days induced involution of the thymus, a positive control observation for in vivo steroid activity, resulting in a 58\% decrease in thymus weight when compared with saline-treated mice (Figure 5A). C.S and/or LPS did not significantly alter thymus weight and Dexinduced thymic involution was not altered by either stimulus. Subchronic CS exposure caused a significant increase in total BALF cell numbers (vehicle; $1 \pm 0.19$ vs. CS; $3.6 \pm 0.18 \times 10^{5}$ cells, $p<0.05$ ) that was completely refractory to Dex treatment (Figure 5B). LPS challenge increased total BALF numbers $\left(10.2 \pm 1.1 \times 10^{5}\right.$ cells $)$ in a manner that was reduced by Dex (49\%). CS exposure prior to LPS reduced the efficacy of Dex, where only a $21 \%$ reduction in total cell numbers was observed. BALF macrophages were only increased in CS exposed mice ( $\sim 2$-fold) that was not significantly altered by LPS or Dex treatment (Figure $5 \mathrm{C}$ ).

\section{Neutrophil activation is associated with GC refractory MMP-9 release in vivo}

CS exposure increased BAL neutrophil numbers (vehicle; $0.08 \pm 0.04$ vs. CS; $\left.1.9 \pm 0.21 \times 10^{5}, p<0.05\right)$ in a Dex insensitive

Table 1. COPD patient characteristics.

\begin{tabular}{llllll}
\hline GOLD Criteria & $\mathbf{n}$ & M:F & Age (range) & Pack Years (range) & \% using ICS \\
\hline II & 9 & $5: 4$ & $64(56-72)$ & $40(20-70)$ & $3 / 9(33 \%)$ \\
III & 9 & $6: 3$ & $70(60-75)$ & $35(20-150)$ & $5 / 9(56 \%)$ \\
IV & 11 & $7: 4$ & $74(69-80)$ & $45(20-116)$ & $10 / 11(91 \%)$ \\
\hline
\end{tabular}

\#Total daily ICS dose (beclamethasone equivalents), based on the assumption that $1 \mu \mathrm{g}$.

beclomethasone $=1 \mu \mathrm{g}$ Budesonide $=0.5 \mu \mathrm{g}$ Fluticasone.

doi:10.1371/journal.pone.0033277.t001 
A

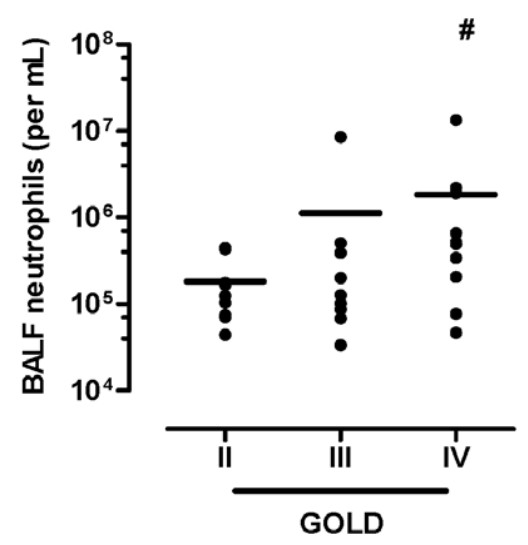

B

C
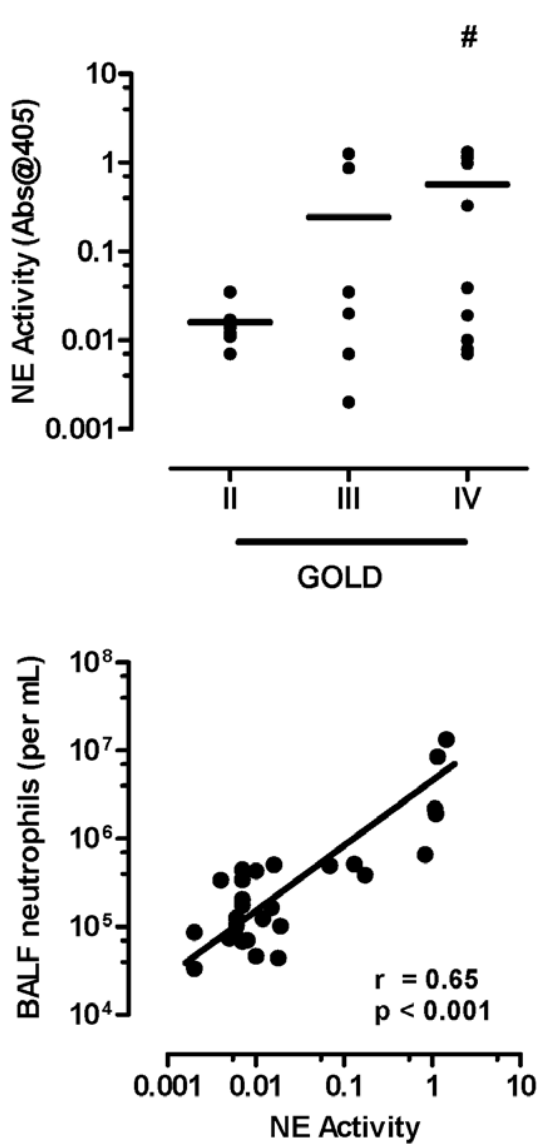

Figure 1. Neutrophilic airway inflammation increases with disease severity. (A) BALF neutrophils were higher in very severe COPD (GOLD IV) compared to participants with moderate COPD (GOLD II). (B) NE activity stratified across the GOLD stages demonstrates increased NE activity in GOLD IV. (C) Neutrophils were positively related to NE activity (Spearman $r=0.65, p<0.001$ ) in BALF. Individual data points are shown with line representing mean values. $\# p<0.05$ versus GOLD II.

doi:10.1371/journal.pone.0033277.g001

manner (Figure 6A). LPS markedly increased BAL neutrophil numbers $\left(9.022 \pm 1.1 \times 10^{5}\right.$ neutrophils $\left./ \mathrm{mL}\right)$ and Dex suppressed this response by $54 \%$. The combination of LPS and CS did not significantly alter the LPS neutrophilic response but did reduce the efficacy of Dex to $23 \%$. NE activity in BALF was not significantly altered by CS, Dex or LPS treatment (Figure 6B). The combination of CS and LPS significantly increased NE activity (vehicle; $100 \pm 15.6$ vs. CS-LPS; $176 \pm 15.6 \%, p<0.05$ ). The response was further increased in the presence of Dex $(222 \pm 17.7 \%)$. MMP-9 expression in BALF was modestly increased in CS exposed mice and insensitive to Dex (Figure 6C). There was a large increase in MMP-9 expression in LPSchallenged mice (vehicle; $30.9 \pm 10.7$ vs. LPS; 33571 \pm 4930 , $p<0.05$ ) and Dex suppressed this response by $89 \%$. CS exposure prior to LPS reduced the efficacy of Dex, where a $42 \%$ reduction in MMP-9 was observed.

\section{Discussion}

The close association between MMP-9 and BALF neutrophils in our COPD cohort identify this cell type as a major source of MMP-9. Increasing MMP-9 and NE with disease severity in the presence of increasing ICS dosage is consistent with enhanced neutrophil activation and degranulation. Our in vitro data demonstrates that fMLP stimulated blood neutrophils release granules containing MMP-9 and NE in a GC resistant manner. Furthermore, degranulation of NE and MMP-9 share similar signalling pathways (PI3K dependence), but diverge in their requirement for MAPK signalling, where only MMP-9 was reduced by Erk and p38 pathway inhibition. Our findings are consistent with previous observations that also identified MAPK (Erk1/2 and p38) [19] signalling as necessary for MMP-9 degranulation. The release of MMP-9 by neutrophils can also occur in response to other inflammatory mediators such as CXCL8 [20], TNF $\alpha$ [21] and endotoxin [22]. Unlike denovo MMP-9 production by other cell types such as macrophages, this response occurs rapidly and is released independently of TIMP1, as neutrophils do not produce this anti-proteinase [10]. MMP-9 is formed in the later stages of neutrophil maturation and this proteinase contributes to neutrophil extravasation and stem cell mobilisation [23,24] via the degradation of basement membrane collagens, whereas NE is primarily responsible for pathogen killing (reviewed in [25]).

Primary azurophilic granules containing NE normally undergo limited exocytosis and are traditionally associated with the intracellular killing of microorganisms in the phagolysosome, as mice deficient in $\mathrm{NE}$ are more susceptible to gram negative bacteria [26]. The majority of NE is expressed on the neutrophil surface during activation, with less than $5 \%$ being released into the extracellular milieu [27]. In COPD, free NE activity may be further increased by necrotic neutrophils. Haemophilus Influenzae is a common gram negative pathogen in COPD that chronically colonises the airways and directly causes neutrophil necrosis and release of azurophilic granular content [28]. In vivo, only C.Sprimed neutrophils release free NE activity in response to LPS challenge in a manner that was completely refractory to GGs. The hierarchy of granular release is only partially understood and involves intracellular calcium changes that can differentially regulate degranulation, where azurophilic granule release is dependent on calcium transient, in contrast to CXCL8-mediated gelatinase exocytosis [29]. Calcium changes regulate soluble- $\mathcal{N}$ ethylmaleimidesensitive-factor accessory-protein receptors (SNAREs), a system responsible for fusion of granules with the plasma membrane [30] and the density of SNAREs may regulate exocytosis potential. Components of CS such as nicotine have been shown to increase intracellular calcium levels in neutrophils [31], however it is not known whether this response further primes azurophilic exocytosis or whether the expression or function of SNAREs are altered with increasing COPD severity.

In vivo, MMP-9 release was maximal in response to LPS challenge in a manner that was effectively blocked by GCs. This occurred in 
A

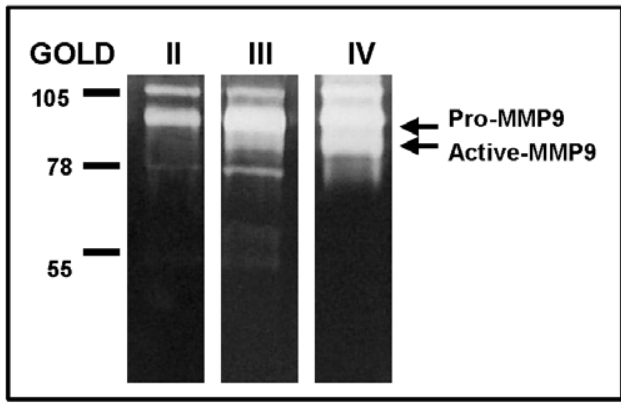

B

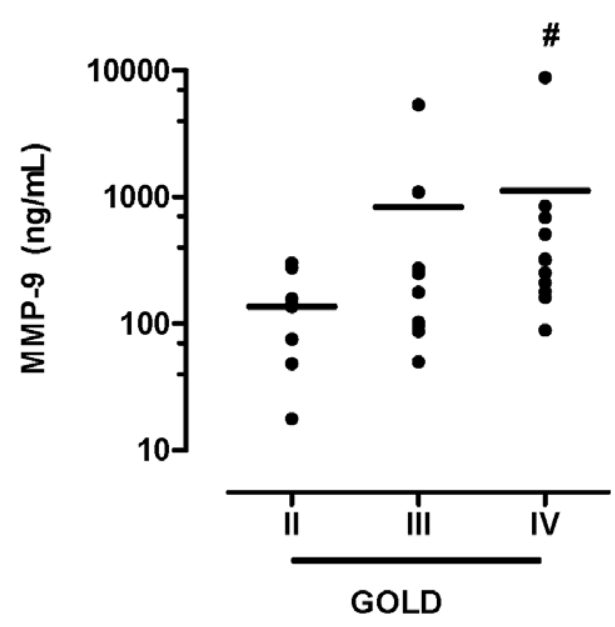

D

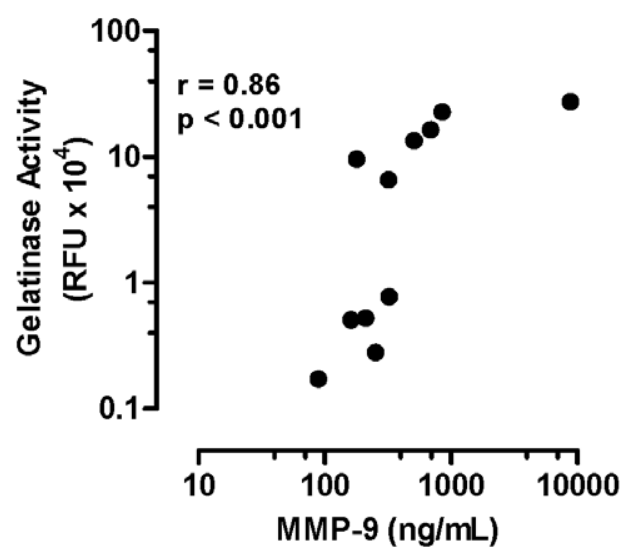

Figure 2. MMP-9 expression and activity increased in severe COPD. (A) Gelatin zymography (representative bands from each GOLD stage) identify major bands of activity correlating to the molecular weight of pro (92 kDa) and active (82 kDa) MMP-9. (B) MMP-9 expression and (C) activity increased with disease severity. (D) MMP-9 expression and gelatinase activity in GOLD IV BALF were strongly related (Spearman $r=0.86, p<0.001)$. Individual data points are shown with line representing mean values. \# $p<0.05$ versus GOLD II.

doi:10.1371/journal.pone.0033277.g002

the absence of neutrophil activation as determined by measuring concurrent NE release. The absence of neutrophil activation suggests that alternative cellular sources are producing MMP-9 in response to LPS alone, such as alveolar macrophages. Alveolar macrophages increase production of MMP-9 in response to LPS stimulation in a GC sensitive manner [32], which is consistent with our in vivo data. In COPD, epigenetic deregulation in COPD macrophages is associated with oxidant dependant loss of HDAC2 expression and subsequent loss of GC mediated suppression of inflammatory genes [33]. In addition, our data support neutrophil activation of as an alternative mechanism for GC refractoryMMP-9 release. We found that C.S priming and LPS challenge promoted neutrophil activation and this was associated with a reduction in GC efficacy to block MMP-9 in vivo. This data suggests that there is a transition from macrophage to neutrophil mediated release of MMP-9 in the presence of GCs and is consistent with our in vitro findings where neutrophil derived MMP-9 was completely resistant to GCs. Reduced Dex actions may also initiate a positive feedback inflammatory loop, as matrix breakdown products further augment neutrophil release of MMP-9 [11].
Elevated NE levels may also directly contribute to ongoing inflammation. NE activates TLR-4 signalling to promote CXCL8 expression in bronchial epithelial cells via activation of $\mathrm{NF \kappa B}$ $[16,34]$. Whether NE directly engages TLR-4 or modifies a required surface co-receptor or adaptor molecule has yet to be elucidated. In addition, NE was identified at the apex of a signalling cascade that can promote CXCL8 release via EGFR signalling in bronchial epithelial cells [35]. This process involves the surface shedding of EGFR ligands that initiate receptor transactivation and subsequent activation of $\mathrm{p} 38$ and NFkB. EGFR transactivation is also implicated in other pathological processes in COPD. NE mediated activation of this pathway promotes airway mucin production [36]. Furthermore, we have previously shown that EGFR transactivation augments inflammatory responses initiated by rhinovirus infection of bronchial epithelial cells [37].

In summary, we found that both NE and MMP-9 activity was elevated in severe COPD and was closely related to neutrophilic inflammation. In vivo, neutrophil activation was associated with GC refractory release of MMP-9. In vitro, neutrophils rapidly release both proteinases in a GC resistant manner that was dependent on PI3K 
A

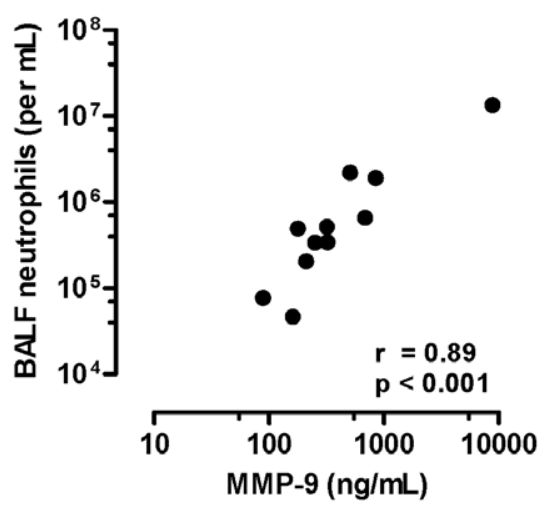

C

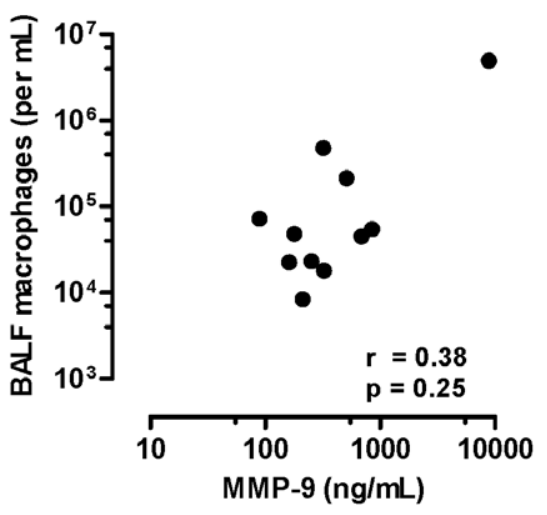

B

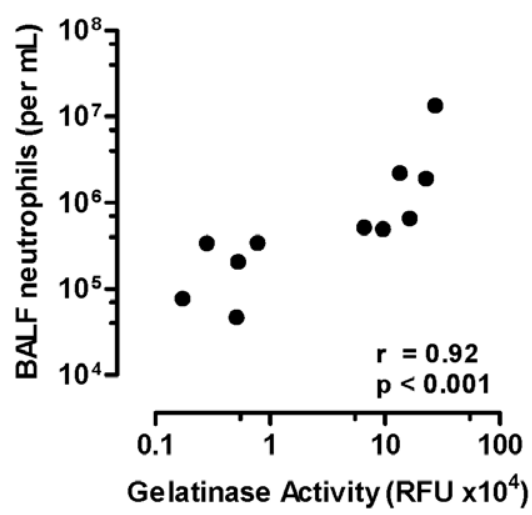

D

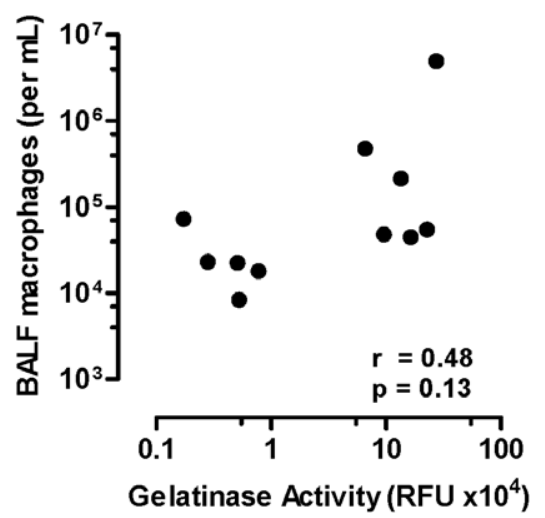

Figure 3. MMP-9 is associated with neutrophilic inflammation in COPD. (A) MMP-9 expression was positively associated with BALF neutrophils in GOLD IV COPD (Spearman $r=0.0 .89, p<0.001$ ). (B) MMP-9 activity was also positively associated with BALF neutrophils in GOLD IV COPD (Spearman $r=0.0 .92, p<0.001$ ), whereas no association was observed with BALF macrophages (C \& D).

doi:10.1371/journal.pone.0033277.g003

signalling. Maximal release of MMP-9, but not NE was also dependent on Erk and p38 signalling. Neutrophil activation may represent a fundamental mechanism of GC resistance in COPD, as $\mathrm{NE}$ is at the apex of inflammatory and mucus pathways and its release is completely resistant to GCs. By identifying the mechanisms for neutrophil derived NE and MMP-9 release in chronic inflammatory conditions and their lack of regulation by currently used antiinflammatory agents such as GCs, better therapeutic strategies can be designed to combat deregulated inflammation in COPD.

\section{Methods}

\section{Ethics Statement}

This research was approved by the Hunter New England Human Research Ethics Committee of the Hunter New England Area Health Service (HNEAH). All participants gave informed written consent prior to their inclusion in this study. The animal experiments were approved by the Animal Ethics Committee (AEC) of the University of Melbourne and conducted in compliance with the National Health and Medical Research Council (NHMRG) of Australia.

\section{Subjects}

Fiberoptic bronchoscopy was performed on subjects with stable COPD $(n=29$; GOLD II $(n=9)$, GOLD III $(n=9)$ and GOLD IV $(n=11)$. Patient characteristics are summarized in Table 1 . The percentage use and daily dosage of ICS at time of sampling is summarized in Table 1 . Warmed saline $(2 \times 60 \mathrm{~mL})$ was instilled into the airways to enable recovery of BAL cells and BAL fluid (BALF). BAL cells were collected by centrifugation $(400 \times g$ for $5 \mathrm{~min}$ ) and cytospot slides were prepared and stained for differential cell analysis based on morphology. Cell free BALF was archived at $-80^{\circ} \mathrm{C}$ for future analysis.

\section{Proteinase Assays}

Measurement of NE activity in BALF and supernatant was determined using the specific NE substrate methoxysuccinylalanyl-alanyl-prolyn-valylpapnitroanalide (MEOSAAPVNA, Sigma; St Louis, MO). Neat BALF ( $5 \mu \mathrm{L}$ ) was diluted to $200 \mu \mathrm{L} \mathrm{NE}$ Buffer containing final concentrations of $0.2 \mathrm{mM}$ MEO-

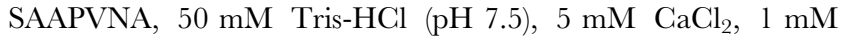
$\mathrm{ZnCl}_{2}$ and $0.01 \%$ sodium azide. Absorbance at $405 \mathrm{~nm}$ was measured using microplate reader (MultiScan Ascent, Thermo).

Zymography was used to quantify gelatinase activity in BALF, which identified major gelatinase bands observed at $\sim 90 \mathrm{kDa}$ corresponding to MMP-9 as previously published [38]. Briefly, gelatin substrate (2 $\mathrm{mg} / \mathrm{mL}$, LabChem Inc, Pittsburgh, PA) was incorporated into SDS polyacrylamide mini-gels (10\%) before casting. BALF $(10 \mu \mathrm{L})$ was run into gels at a constant voltage $(200 \mathrm{~V})$ under non-reducing conditions. Gels were washed twice in 
$\mathbf{A}$

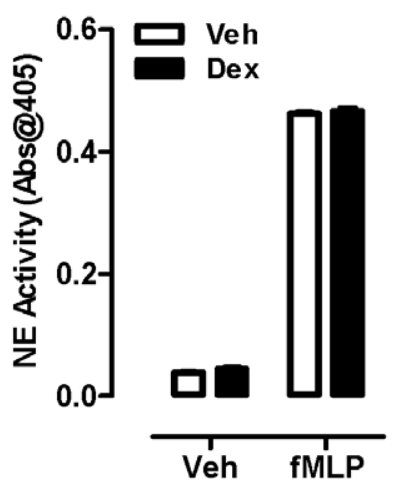

C

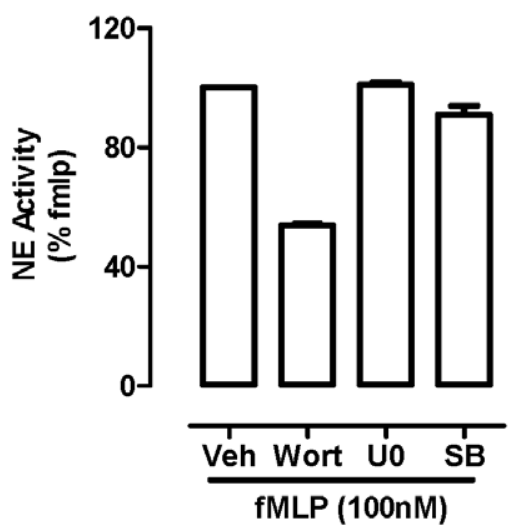

B

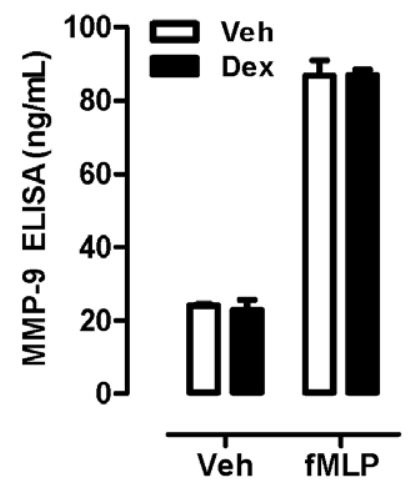

D

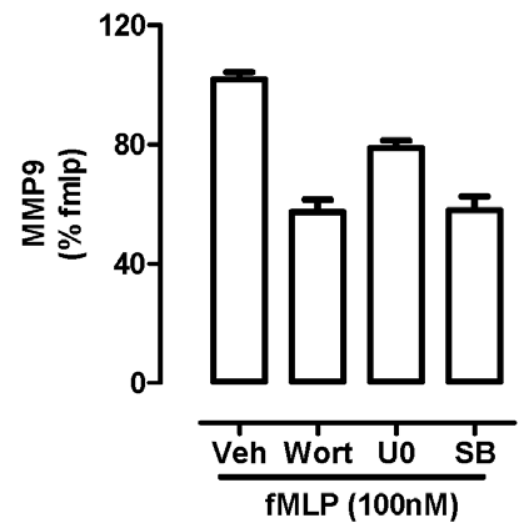

Figure 4. Neutrophils release NE and MMP-9 in a GC resistant manner. Isolated blood neutrophils from healthy volunteers were pretreated with Vehicle (Veh, $0.01 \%$ DMSO) or Dex (100 nM) for 15 min prior to fMLP stimulation (100 nM, 20 min at 37C) and (A) NE activity and (B) MMP-9 release was determined. Isolated neutrophils were also pretreated with wortmannin (Wort, $100 \mathrm{nM}), \mathrm{U} 0126$ (U0, $1 \mu \mathrm{M})$ and SB203580 (SB, $1 \mu \mathrm{M})$ prior to $\mathrm{fMLP}$ stimulation and cell free supernatants were retained for assessment of (C) NE activity and (D) MMP-9 expression as a percentage of the fMLP positive control. Data is presented as the mean \pm SEM of the 3 replicates from an individual donor and is representative of 3 separate experiments. doi:10.1371/journal.pone.0033277.g004

Triton X-100 solution (2.5\%) for 15 minutes and incubated overnight at $37^{\circ} \mathrm{C}$ in zymography buffer $(50 \mathrm{mM}$ Tris-HCl (pH 7.5), $5 \mathrm{mM} \mathrm{CaCl}_{2}, 1 \mathrm{mM} \mathrm{ZnCl}_{2}$ and $0.01 \%$ sodium azide). The gels were then stained with Coomassie Brilliant Blue R-250 (Sigma St Louis, MO) for 45 minutes and extensively destained. After destaining, zones of enzyme activity appeared clear against the background of Coomassie Blue stain.

Total MMP-9 levels (pro and active forms) were assessed using quantitative ELISA (DuoSet ELISA, RnD Systems) in accordance with the manufacturer's instructions. Measurement of MMP-9 activity in BALF and was determined using fluorescein labeled DQ gelatin substrate (Life Technologies), which is only cleaved by the active form of the enzyme. Neat BALF $(40 \mu \mathrm{L})$ was diluted to $200 \mu \mathrm{L}$ Buffer containing final concentrations of $3 \mu \mathrm{g}$ fluorescein labeled DQ gelatin substrate, $50 \mathrm{mM}$ Tris-HCl (pH 7.5), $5 \mathrm{mM}$ $\mathrm{CaCl}_{2}, 1 \mathrm{mM} \mathrm{ZnCl}_{2}$ and $0.01 \%$ sodium azide. Fluorescence intensity (relative fluorescence units, RFU) was measured using a fluoresecence microplate reader with standard fluorescein filters. Digested products from DQ Gelatin have absorption maxima of $495 \mathrm{~nm}$ and emission maxima of $515 \mathrm{~nm}$.

\section{In vitro Analysis}

All reagents were obtained from Sigma-Aldrich unless otherwise stated. Venous blood was collected upon receipt of written consent from healthy volunteers. Neutrophils were isolated by Ficoll-Paque density gradient centrifugation. The pellet enriched for erythrocytes and neutrophils was next mixed with an equal volume of $6 \%$ Dextran T500 in saline and allowed to sediment for $20 \mathrm{~min}$. The neutrophil enriched upper layer was removed and contaminating erythrocytes were removed by hypotonic lysis. Cell viability was checked using Trypan blue exclusion method and was routinely found to be $>95 \%$. Neutrophil purity was confirmed by differential staining to be $>95 \%$. Neutrophils were resuspended to $2 \times 10^{6}$ cells per $\mathrm{mL}$ in Krebs Buffer and incubated with $5 \mu \mathrm{g} / \mathrm{mL}$ Cytochalasin B for $15 \mathrm{~min}$ prior to stimulation. Isolated cells were also pretreated with Dex $(100 \mathrm{nM})$, wortmannin $(100 \mathrm{nM})$, U0126 $(1 \mu \mathrm{M})$ or SB203580 $(1 \mu \mathrm{M})$ at $37 \mathrm{C}$ for $15 \mathrm{~min}$ prior to fMLP stimulation $(100 \mathrm{nM}, 37 \mathrm{C}$ for $20 \mathrm{~min})$. At the end of the incubation, cell free supernatant was retained by centrifugation at $1000 \mathrm{~g}$ for $5 \mathrm{~min}$ and archived at $-80 \mathrm{C}$ prior to NE and MMP9 analysis. 
A

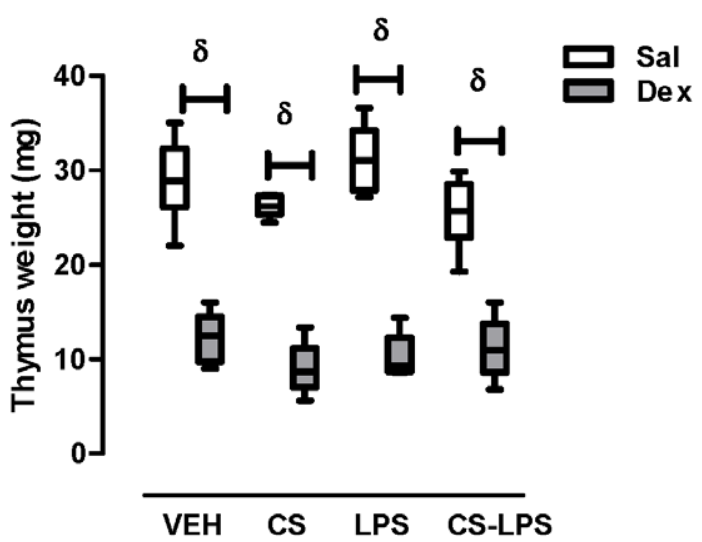

B

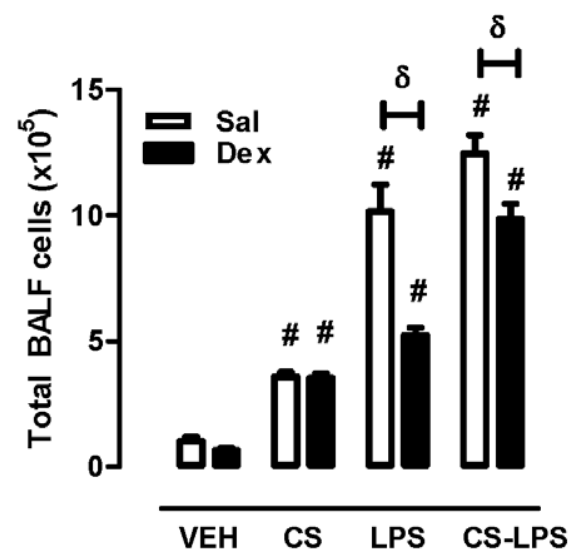

C

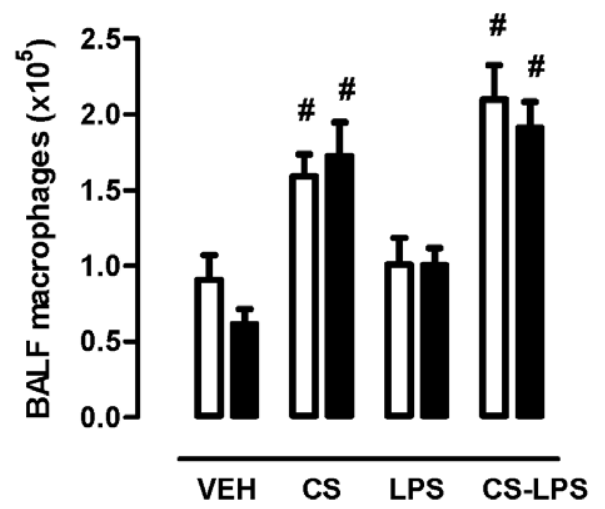

Figure 5. CS exposure reduces the efficacy of Dex to suppress acute airway inflammation. Mice were treated with Saline (open bar) or Dex (closed bar, $1 \mathrm{mg} / \mathrm{kg}$ i.p) once daily over 4 days for 2 hours prior to CS exposure as detailed in methods. On Day 4, mice were treated intranasally with saline (VEH) or LPS $(1 \mu \mathrm{g})$. Following 24 hour LPS challenge mice were sacrificed and (A) thymus weight was recorded. BAL was also performed and (B) total cell and (C) macrophages numbers were determined by differential cell counting analysis. $\delta$ Two-way ANOVA (Sal versus Dex, $p<0.05$ ). \# One-way ANOVA (versus VEH, $p<0.05$ ).

doi:10.1371/journal.pone.0033277.g005

\section{In vivo Analysis}

Specific pathogen-free male Balb/c mice aged 7 weeks and weighing $\sim 20 \mathrm{~g}$ were obtained from the Animal Resource Centre Pty. Ltd. (Perth, Australia). The animals were housed at $20^{\circ} \mathrm{C}$ on a

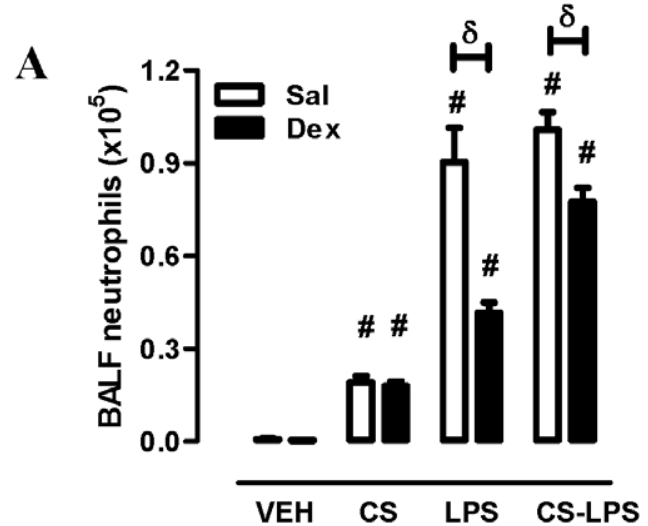

B

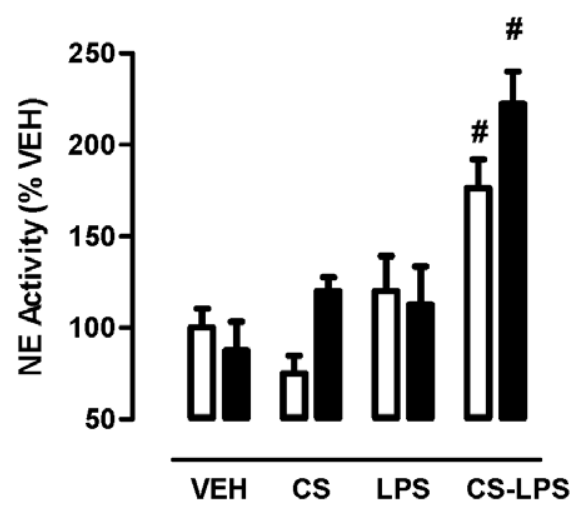

C

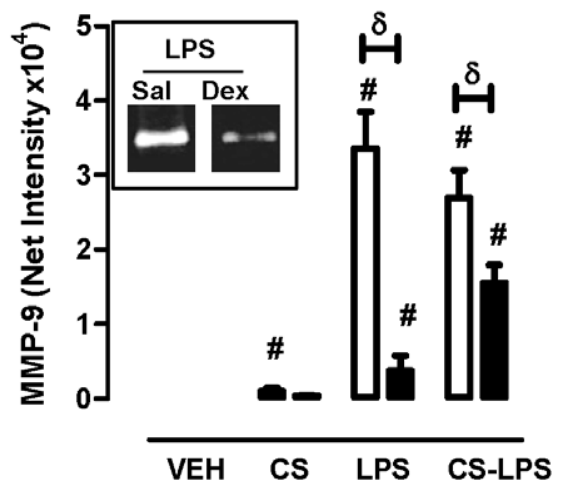

Figure 6. Differential MMP-9 and NE activity and altered GS responses in vivo. (A) BALF neutrophil numbers were determined from treatment groups as detailed in Figure 5 legend. BALF was retained and (B) NE activity and (C) MMP-9 expression (Insert: representative zymography results) was determined as detailed in methods (C) $\delta$ Two-way ANOVA (Sal versus Dex, $p<0.05$ ). \# One-way ANOVA (versus VEH, $p<0.05$ ). The data was pooled from 2 separate experiments ( $n=5-10$ mice per treatment group). doi:10.1371/journal.pone.0033277.g006

12-h day/night cycle in sterile micro-isolators and fed a standard sterile diet. Mice were treated with Dex ( $1 \mathrm{mg} / \mathrm{kg}$, i.p.) for 2 hours prior to CS exposure. For the sub-chronic CS exposure protocols, mice were placed in an 18 liter perspex chamber in a class II biosafety cabinet as previously described [39] with some minor 
modifications. Briefly, mice were exposed to whole body sidestream CS generated from one cigarette (2R4F, University of Kentucky, Lexington, KY) for 15 min and allowed to recover for 2 hours. The levels of carboxyhemoglobin in the blood of Balb/c mice exposed to CS using this model have previously been measured and found to be comparable to that observed in smokers [39]. This process was repeated 2 times for a total of 3 cigarettes per day over four days. On the fourth day, the final CS exposure was replaced by treating the mice with LPS. For LPS challenge, mice were anesthetised by methoxyflurane (Medical Developments International Ltd, Springvale, Australia) inhalation and treated intranasally with $1 \mu \mathrm{g}$ LPS diluted in $50 \mu \mathrm{L}$ PBS. Saline was used as a control for Dex and LPS. Following 24 hours, thymus weight was also recorded as a quantitative measure of GC activity. BAL was also performed via tracheotomy (SP30 Duran polyethylene tubing) proximal to the larynx and total/differential BAL cell counts were performed as previously described [40]. Cell free BALF was archived at $-80^{\circ} \mathrm{C}$ prior to analysis of MMP-9 and NE activity.

\section{References}

1. Taggart CC, Greene CM, Carroll TP, O’Neill SJ, McElvaney NG (2005) Elastolytic proteases: inflammation resolution and dysregulation in chronic infective lung disease. Am J Respir Crit Care Med 171: 1070-1076.

2. Owen CA (2008) Roles for proteinases in the pathogenesis of chronic obstructive pulmonary disease. Int J Chron Obstruct Pulmon Dis 3: 253-268.

3. Pauwels RA, Rabe KF (2004) Burden and clinical features of chronic obstructive pulmonary disease (COPD). Lancet 364: 613-620.

4. Donaldson GC, Seemungal TA, Patel IS, Lloyd-Owen SJ, Wilkinson TM, et al. (2003) Longitudinal changes in the nature, severity and frequency of COPD exacerbations. Eur Respir J 22: 931-936.

5. Sethi S, Murphy TF (2008) Infection in the pathogenesis and course of chronic obstructive pulmonary disease. N Engl J Med 359: 2355-2365.

6. Culpitt SV, Maziak W, Loukidis S, Nightingale JA, Matthews JL, et al. (1999) Effect of high dose inhaled steroid on cells, cytokines, and proteases in induced sputum in chronic obstructive pulmonary disease. Am J Respir Crit Care Med 160: 1635-1639.

7. Beeh KM, Beier J, Kornmann O, Buhl R (2003) Sputum matrix metalloproteinase-9, tissue inhibitor of metalloprotinease-1, and their molar ratio in patients with chronic obstructive pulmonary disease, idiopathic pulmonary fibrosis and healthy subjects. Respir Med 97: 634-639.

8. Culpitt SV, Rogers DF, Traves SL, Barnes PJ, Donnelly LE (2005) Sputum matrix metalloproteases: comparison between chronic obstructive pulmonary disease and asthma. Respir Med 99: 703-710.

9. Faurschou M, Borregaard N (2003) Neutrophil granules and secretory vesicles in inflammation. Microbes Infect 5: 1317-1327.

10. Masure S, Proost P, Van Damme J, Opdenakker G (1991) Purification and identification of $91-\mathrm{kDa}$ neutrophil gelatinase. Release by the activating peptide interleukin-8. Eur J Biochem 198: 391-398.

11. Xu X, Jackson PL, Tanner S, Hardison MT, Abdul Roda M, et al. (2011) A selfpropagating matrix metalloprotease-9 (MMP-9) dependent cycle of chronic neutrophilic inflammation. PLoS One 6: e15781.

12. Damiano VV, Tsang A, Kucich U, Abrams WR, Rosenbloom J, et al. (1986) Immunolocalization of elastase in human emphysematous lungs. J Clin Invest 78: 482-493.

13. Laurell CB, Eriksson S (1963) The electrophoretic alpha-1-globulin pattern of serum in alpha-1-antitrypsin defi ciency. Scand J Clin Lab Invest. pp 132-140.

14. Ferry G, Lonchampt M, Pennel L, de Nanteuil G, Canet E, et al. (1997) Activation of MMP-9 by neutrophil elastase in an in vivo model of acute lung injury. FEBS Lett 402: 111-115.

15. Itoh Y, Nagase H (1995) Preferential inactivation of tissue inhibitor of metalloproteinases-1 that is bound to the precursor of matrix metalloproteinase 9 (progelatinase B) by human neutrophil elastase. J Biol Chem 270: 16518-16521.

16. Devaney JM, Greene CM, Taggart CG, Carroll TP, O’Neill SJ, et al. (2003) Neutrophil elastase up-regulates interleukin-8 via toll-like receptor 4. FEBS Lett 544: 129-132.

17. Yan C, Wang H, Aggarwal B, Boyd DD (2004) A novel homologous recombination system to study $92 \mathrm{kDa}$ type IV collagenase transcription demonstrates that the NF-kappaB motif drives the transition from a repressed to an activated state of gene expression. FASEB J 18: 540-541.

18. Bozinovski S, Hutchinson A, Thompson M, Macgregor L, Black J, et al. (2008) Serum amyloid a is a biomarker of acute exacerbations of chronic obstructive pulmonary disease. Am J Respir Crit Care Med 177: 269-278.

\section{Statistical analysis}

Normally distributed data were expressed as mean \pm S.E. For the clinical samples Mann Whitney U test was used to analyze the data. Spearman correlation was used to assess the relationship between secreted proteinases and neutrophil numbers in BALF and the relationship between MMP-9 and NE. For the animal studies, significance between groups was tested using either oneway or two-way analysis of variance (ANOVA) followed by Dunnetts comparison test where $\mathrm{p} \leq 0.05$ was considered significant.

\section{Acknowledgments}

The authors wish to acknowledge the technical contributions of Huei Junn Seow, Andrew Lilja and Lovisa Dousha.

\section{Author Contributions}

Conceived and designed the experiments: SB RV GPA PW. Performed the experiments: SB RV PW. Analyzed the data: SB RV PW GPA. Contributed reagents/materials/analysis tools: PW. Wrote the paper: SB RV GPA PW.

19. Ehrenfeld P, Matus CE, Pavicic F, Toledo C, Nualart F, et al. (2009) Kinin B1 receptor activation turns on exocytosis of matrix metalloprotease- 9 and myeloperoxidase in human neutrophils: involvement of mitogen-activated protein kinase family. J Leukoc Biol 86: 1179-1189.

20. Opdenakker G, Van den Steen PE, Dubois B, Nelissen I, Van Coillie E, et al. (2001) Gelatinase B functions as regulator and effector in leukocyte biology. J Leukoc Biol 69: 851-859.

21. Chakrabarti S, Zee JM, Patel KD (2006) Regulation of matrix metalloproteinase-9 (MMP-9) in TNF-stimulated neutrophils: novel pathways for tertiary granule release. J Leukoc Biol 79: 214-222.

22. Pugin J, Widmer MC, Kossodo S, Liang CM, Preas HLn, et al. (1999) Human neutrophils secrete gelatinase $\mathrm{B}$ in vitro and in vivo in response to endotoxin and proinflammatory mediators. Am J Respir Cell Mol Biol 20: 458-464.

23. Opdenakker G, Fibbe WE, Van Damme J (1998) The molecular basis of leukocytosis. Immunol Today 19: 182-189.

24. Pruijt JF, Fibbe WE, Laterveer L, Pieters RA, Lindley IJ, et al. (1999) Prevention of interleukin-8-induced mobilization of hematopoietic progenitor cells in rhesus monkeys by inhibitory antibodies against the metalloproteinase gelatinase B (MMP-9). Proc Natl Acad Sci U S A 96: 10863-10868.

25. Pham CT (2006) Neutrophil serine proteases: specific regulators of inflammation. Nat Rev Immunol 6: 541-550.

26. Belaaouaj A, McCarthy R, Baumann M, Gao Z, Ley TJ, et al. (1998) Mice lacking neutrophil elastase reveal impaired host defense against gram negative bacterial sepsis. Nat Med 4: 615-618.

27. Owen CA, Campbell MA, Sannes PL, Boukedes SS, Campbell EJ (1995) Cell surface-bound elastase and cathepsin G on human neutrophils: a novel, nonoxidative mechanism by which neutrophils focus and preserve catalytic activity of serine proteinases. J Cell Biol 131: 775-789.

28. Naylor EJ, Bakstad D, Biffen M, Thong B, Calverley P, et al. (2007) Haemophilus influenzae induces neutrophil necrosis: a role in chronic obstructive pulmonary disease? Am J Respir Cell Mol Biol 37: 135-143.

29. Chakrabarti S, Patel KD (2005) Regulation of matrix metalloproteinase-9 release from IL-8-stimulated human neutrophils. J Leukoc Biol 78: 279-288.

30. Salaun C, James DJ, Greaves J, Chamberlain LH (2004) Plasma membrane targeting of exocytic SNARE proteins. Biochim Biophys Acta 1693: 81-89.

31. Ryder MI (1994) Nicotine effects on neutrophil F-actin formation and calcium release: implications for tobacco use and pulmonary diseases. Exp Lung Res 20: 283-296.

32. Russell RE, Culpitt SV, DeMatos G, Donnelly L, Smith M, et al. (2002) Release and activity of matrix metalloproteinase-9 and tissue inhibitor of metalloproteinase-1 by alveolar macrophages from patients with chronic obstructive pulmonary disease. Am J Respir Cell Mol Biol 26: 602-609.

33. Barnes PJ, Ito K, Adcock IM (2004) Corticosteroid resistance in chronic obstructive pulmonary disease: inactivation of histone deacetylase. Lancet 363 : $731-733$.

34. Walsh DE, Greene CM, Carroll TP, Taggart CC, Gallagher PM, et al. (2001) Interleukin-8 up-regulation by neutrophil elastase is mediated by MyD88/ IRAK/TRAF-6 in human bronchial epithelium. J Biol Chem 276: 35494-35499.

35. Kuwahara I, Lillehoj EP, Lu W, Singh IS, Isohama Y, et al. (2006) Neutrophil elastase induces IL-8 gene transcription and protein release through p38/NF\{kappa\}B activation via EGFR transactivation in a lung epithelial cell line. Am J Physiol Lung Cell Mol Physiol 291: L407-416. 
36. Shao MX, Nadel JA (2005) Neutrophil elastase induces MUC5AC mucin production in human airway epithelial cells via a cascade involving protein kinase $\mathrm{C}$, reactive oxygen species, and $\mathrm{TNF}$-alpha-converting enzyme. J Immunol 175: 4009-4016.

37. Liu K, Gualano RC, Hibbs ML, Anderson GP, Bozinovski S (2008) Epidermal growth factor receptor signaling to Erk1/2 and STATs control the intensity of the epithelial inflammatory responses to rhinovirus infection. J Biol Chem 283: 9977-9985.

38. Bozinovski S, Jones JE, Vlahos R, Hamilton JA, Anderson GP (2002) Granulocyte/macrophage-colony-stimulating factor (GM-CSF) regulates lung innate immunity to lipopolysaccharide through Akt/Erk activation of NFkappa B and AP-1 in vivo. J Biol Chem 277: 42808-42814.

39. Vlahos R, Bozinovski S, Jones JE, Powell J, Gras J, et al. (2006) Differential protease, innate immunity, and NF-kappaB induction profiles during lung inflammation induced by subchronic cigarette smoke exposure in mice. Am J Physiol Lung Cell Mol Physiol 290: L931-945.

40. Bozinovski S, Cross M, Vlahos R, Jones JE, Hsuu K, et al. (2005) S100A8 chemotactic protein is abundantly increased, but only a minor contributor to LPS-induced, steroid resistant neutrophilic lung inflammation in vivo. J Proteome Res 4: 136-145. 